\title{
Elevated lung clearance index in infants with cystic fibrosis shortly after birth
}

\author{
Elisabeth Kieninger ${ }^{1,10}$, Sophie Yammine ${ }^{1,10}$, Insa Korten ${ }^{1,2}$, \\ Pinelopi Anagnostopoulou ${ }^{1}$, Florian Singer ${ }^{1,3}$, Urs Frey $^{2}$, Anne Mornand ${ }^{4}$, \\ Maura Zanolari ${ }^{5}$, Isabelle Rochat ${ }^{6}$, Daniel Trachsel ${ }^{2}$, Dominik Mueller-Suter ${ }^{7}$, \\ Alexander Moeller ${ }^{3}$, Carmen Casaulta ${ }^{1}$, Philipp Latzin ${ }^{1}$ and the SCILD ${ }^{8}$ and \\ BILD study groups ${ }^{9}$
}

\begin{abstract}
Affiliations: 'Paediatric Respiratory Medicine, Inselspital, University Children's Hospital of Bern, University of Bern, Bern, Switzerland. ${ }^{2}$ Dept of Paediatrics, University Children's Hospital of Basel, Basel, Switzerland. ${ }^{3}$ Division of Respiratory Medicine, University Children's Hospital of Zurich, Zurich, Switzerland. ${ }^{4}$ Dept of the Child and Adolescent, Children's University Hospital of Geneva, Geneva, Switzerland. ${ }^{5}$ Dept of Paediatrics, Hospital of Bellinzona, Bellinzona, Switzerland. 'Paediatric Pulmonology Unit, Department of Paediatrics, CHUV Lausanne, University Hospital of Lausanne, Lausanne, Switzerland. ${ }^{7}$ Dept of Paediatrics, Katonsspital Aarau, Aarau, Switzerland. ${ }^{8}$ Members of the Swiss Cystic Fibrosis Infant Lung Development (SCILD) cohort, current study group can be found in the Acknowledgements section. ${ }^{9}$ Members of the Basel Bern Infant Lung Development (BILD) cohort, current study group can be found in the Acknowledgements section. ${ }^{10}$ Both authors contributed equally to this work.
\end{abstract}

Correspondence: Philipp Latzin, Paediatric Respiratory Medicine, University Children's Hospital of Bern, University of Bern, 3010 Bern, Switzerland. E-mail: philipp.latzindinsel.ch

@ERSpublications

Altered lung function in infants with cystic fibrosis shortly after birth http://ow.ly/qPxq30eFOkW

Cite this article as: Kieninger E, Yammine S, Korten I, et al. Elevated lung clearance index in infants with cystic fibrosis shortly after birth. Eur Respir J 2017; 50: 1700580 [https://doi.org/10.1183/13993003.005802017].

ABSTRACT It is not known at what age lung function impairment may arise in children with cystic fibrosis (CF). We assessed lung function shortly after birth in infants with CF diagnosed by newborn screening.

We performed infant lung function measurements in a prospective cohort of infants with $\mathrm{CF}$ and healthy controls. We assessed lung clearance index (LCI), functional residual capacity (FRC) and tidal breathing parameters. The primary outcome was prevalence and severity of abnormal lung function $( \pm 1.64$ z-scores) in CF.

We enrolled 53 infants with CF (mean age 7.8 weeks) and 57 controls (mean age 5.2 weeks). Compared to controls, LCI and FRC were elevated (mean difference $0.30,95 \%$ CI $0.02-0.60 ; \mathrm{p}=0.034$ and $14.5 \mathrm{~mL}$, 95\% CI 7.7-21.3 mL; $\mathrm{p}<0.001$, respectively), while ratio of time to peak tidal expiratory flow to expiratory time was decreased in infants with CF. In 22 (41.5\%) infants with CF, either LCI or FRC exceeded 1.64 z-scores; three infants had both elevated LCI and FRC.

Shortly after birth, abnormal lung function is prevalent in CF infants. Ventilation inhomogeneity or hyperinflation may serve as noninvasive markers to monitor CF lung disease and specific treatment effects, and could thus be used as outcome parameters for future intervention studies in this age group. 


\section{Introduction}

Newborn screening has introduced a new era of modalities to monitor, treat and improve the prognosis of cystic fibrosis (CF) lung disease [1]. Chest imaging has revealed structural and functional lung abnormalities, such as bronchiectasis and air trapping, even in asymptomatic infants with CF as young as 3-4 months $[2,3]$. This knowledge, in combination with new therapeutic agents [4] that potentially could be used as preventive therapy from infancy onwards [5], has encouraged research to identify noninvasive biomarkers of early CF lung disease. These markers may guide existing therapeutic protocols [6], as well as clinical trials of substances that are potentially disease-modifying [7].

In this regard, multiple-breath inert gas washout $(\mathrm{MBW})$ is a promising lung function measurement technique. It requires only tidal breathing and is feasible from infancy onwards. By measuring the efficiency of the lungs to clear out a marker gas, MBW depicts ventilation distribution inhomogeneity in the lungs [8], with the lung clearance index (LCI) being the most commonly reported outcome [9]. As peripheral CF lung pathology results in ventilation inhomogeneity early in the course of the disease [10], before any change in spirometry [11], LCI is considered the most sensitive lung function marker of mild CF lung disease [12].

LCI values have been reported in infants and small children with CF using sulfur hexafluoride (SF $)$ as an inert gas [13-19]. These studies assessed lung function in infants that were aged $\geqslant 3$ months at the time of the measurement. Some of them used non-commercially available devices, e.g. mass spectrometer [17-19], making wide application almost impossible. Moreover, most of the measurements were performed under sedation [13, 15-19]. Although safe, there is no proof that sedation does not affect breathing pattern $[20,21]$, and it is not known whether this is different in CF and controls.

In this study we hypothesised that lung function during natural sleep may be impaired in infants with $\mathrm{CF}$ diagnosed by newborn screening shortly after birth. We measured MBW and tidal breathing indices in a prospective cohort of mainly asymptomatic infants with $\mathrm{CF}$ and healthy controls. The primary outcome was prevalence and severity of abnormal lung function.

\section{Methods}

\section{Study population}

In this prospective, cross-sectional, observational study we performed lung function measurements in infants with confirmed CF and healthy infants. Infants were assessed between February 2011 and May 2016 at the children's university hospitals of Bern and Basel (Switzerland). All infants with CF were diagnosed by newborn screening [22] and were part of the Swiss Cystic Fibrosis Infant Lung Development Study (SCILD) cohort [23]. Healthy term-born infants were enrolled from the Basel-Bern infant lung development (BILD) cohort [24]. Inclusion criteria were age between 3 and 14 weeks, lack of respiratory symptoms suggesting acute respiratory tract infection as sign of an exacerbation on the day of lung function measurement for infants with CF and no respiratory tract infection within the past 3 weeks prior to lung function measurement in healthy infants. The study was approved by the ethics committee of the Canton Bern and Basel, Switzerland. Parents or caregivers provided written informed consent. For more detailed information, see the online supplementary material.

\section{Lung function measurement}

Lung function measurements were performed via an infant face mask during natural, non-rapid eye movement sleep (by monitoring behaviour and online flow-volume curves) in supine position with the head in midline according to consensus [9] and as reported previously [25]. $10 \mathrm{~min}$ of tidal breathing were recorded, followed by MBW measurements in healthy infants. For reasons of priority, infants with CF first performed MBW followed by tidal breathing. We aimed for three valid MBW tests. Flow was measured using a mainstream ultrasonic flowmeter (Exhalyzer D; Eco Medics, Duernten, Switzerland).

\section{Multiple-breath washout}

Lung volume and ventilation heterogeneity were measured using the $\mathrm{MBW}$ technique using $4 \% \mathrm{SF}_{6}$ as described previously [26]. Main outcomes were functional residual capacity (FRC) and LCI (cumulative expired volume/FRC). Measurements were analysed using software provided by the manufacturer

Support statement: The work for this report was funded by the Swiss Society for Cystic Fibrosis (CFCH), the Gottfried und Julia Bangerter-Rhyner-Stiftung, the Fondazione Ettore e Valeria Rossi, the Swiss National Science Foundation (grant 144280 and 163311 to U. Frey, P. Latzin and C. Kuehni) and the Botnar Foundation. The funders had no role in study design, data collection and analysis, decision to publish, or preparation of the manuscript. Funding information for this article has been deposited with the Crossref Funder Registry.

Conflict of interest: Disclosures can be found alongside this article at erj.ersjournals.com 
(WBreath Version 3.28.0.0; ndd Medizintechnik, Zurich, Switzerland) with a recently validated improved algorithm [25, 27]. We included all valid measurements according to consensus [9] and compared mean test values per child. We recorded all trials and excluded trials with leaks, sighs, arousals, movements and technical reasons. Details on successful and unsuccessful trials as well as reason for exclusions are provided in online supplementary table S1.

To prove the validity of our findings we performed the following sensitivity analyses. 1) Using wash-in traces of the children; 2) using alternative custom-made software (LungSim 2.10; NM, Thalwil, Switzerland; running on MATLAB; MathWorks, Natick, MA, USA) for analysis of washout and wash-in traces [28]; and 3) performing standard analysis in all children with at least two valid measurements.

\section{Tidal breathing}

We used 100 regular breaths of tidal breathing and calculated mean tidal breathing parameters of flow and volume according to current guidelines [24] and as described previously [26]. Outcomes were tidal volume per body weight, respiratory rate, peak inspiratory and expiratory flow and the time to peak tidal expiratory flow ( $t \mathrm{PTEF}$ )/expiratory time ( $\mathrm{tE}$ ) ratio, the latter describing the shape of the tidal breathing flow-volume loop.

\section{Statistical analysis}

Sample size calculation was based on mean \pm SD LCI of $6.75 \pm 0.57$ from 201 healthy term-born infants measured using a similar setup [26]. A sample size of 21 infants in each group would provide $90 \%$ power to detect differences in washout parameter of one population standard deviation between the index and healthy control groups, at a two-sided 5\% significance level. To account for greater variability of tidal breathing parameters and possible drop-out, we aimed to enrol $\geqslant 40$ children. Continuous variables were compared using t-tests after verification of normal distribution. Categorical variables were compared using the Chi-squared test. Upper limit of normal (ULN) was defined as mean+1.64 sD from healthy infants [29] and used to estimate prevalence and severity of abnormal lung function in CF. In all infants with three valid MBW tests, we assessed the intratest variability (coefficient of variation) for LCI and FRC. We examined the relationship between washout parameters, intratest variability and tidal breathing parameters in uni- and multivariable regression analysis. The multivariable model was adjusted for age, body size and sex. Furthermore, we compared the relationship between lung volume (tidal volume and FRC) and body size separately in CF and healthy infants in a post hoc approach by linear regression analysis (for details, see the online supplementary material). All analyses were performed using Stata statistical software (release 11; StataCorp, College Station, TX, USA).

\section{Results}

Study population

We enrolled 54 infants with CF and 57 healthy infants. One infant with CF achieved no successful measurement, leaving 110 infants for analysis. For demographic details and clinical characteristics of patients with CF see tables 1 and 2 and online supplementary table S2. Mean (range) age at lung function measurement was 7.8 (3.6-13.4) weeks in infants with CF and 5.2 (4.1-6.3) weeks in healthy infants, resulting in a mean difference of 2.7 weeks $(95 \% \mathrm{CI}-3.3--2.0$ weeks, $\mathrm{p}<0.001)$. After adjustment for age and sex, infants with CF were significantly lighter and shorter than the healthy infants (table 1) [17]. Three (6\%) CF infants experienced respiratory symptoms prior to lung function measurements; one of them had antibiotic treatment (table 2). Nine (17\%) CF infants were on antibiotic treatment prior to or at the date of the lung function measurements, and 20 (38\%) CF infants were started on regular therapy of inhalative salbutamol and isotonic saline solution prior to the study date (table 2). Lung function measurement was performed $\geqslant 3 \mathrm{~h}$ after inhalation therapy.

\section{Multiple-breath washout}

The mean \pm SD LCI of $7.08 \pm 0.96$ in infants with CF was elevated compared to in healthy controls (6.78 \pm 0.43 ; mean difference $0.30,95 \%$ CI $0.02-0.60$; $\mathrm{p}=0.034) .10$ (19\%) out of 53 infants with CF showed an LCI above the ULN (figure 1, table 3). In addition, FRC was significantly higher in infants with CF (mean difference $14.5 \mathrm{~mL}, 95 \%$ CI $7.7-21.3 \mathrm{~mL}$; $<<0.001$; figure 2), as was FRC per $\mathrm{kg}$ body weight $\left(2.9 \mathrm{~mL} \cdot \mathrm{kg}^{-1}, 1.3-4.4 \mathrm{~mL} \cdot \mathrm{kg}^{-1} ; \mathrm{p}<0.001\right)$ and per $\mathrm{cm}$ body length $\left(0.24 \mathrm{~mL} \cdot \mathrm{cm}^{-1}, 0.13-0.35 \mathrm{~mL} \cdot \mathrm{cm}^{-1}\right.$; $\mathrm{p}<0.001$ ). In 16 (30\%) infants with CF, FRC was above the ULN (figure 2, table 3). Three (5.6\%) infants with CF showed elevated values for both LCI and FRC. One patient with CF presented an outstandingly elevated LCI of 11.94, representing a true physiological outlier and was therefore included in the analysis. Results excluding this physiological outlier are presented in online supplementary table S3.

Sensitivity analysis of wash-in traces and using alternative software for analysis of washout and wash-in traces all confirmed significantly elevated LCI and FRC in infants with CF compared to healthy controls 


\begin{tabular}{|c|c|c|c|c|}
\hline & Healthy & Cystic fibrosis & Mean difference $(95 \% \mathrm{CI})$ & p-value $\#$ \\
\hline Subjects (male) & $57(31)$ & $53(31)$ & & 0.66 \\
\hline Birthweight kg & $3.43 \pm 0.50$ & $3.20 \pm 0.42$ & $-0.23(-0.40--0.05)$ & 0.012 \\
\hline Age weeks & $5.2 \pm 0.6$ & $7.8 \pm 2.4$ & $2.7(2.0-3.3)$ & $<0.001$ \\
\hline Height cm & $54.7 \pm 2.3$ & $55.2 \pm 2.9$ & $0.5(-0.5-1.5)$ & 0.32 \\
\hline Height centiles & $48.80 \pm 30.64$ & $27.05 \pm 23.62$ & $-21.75(-32.14--11.35)$ & $<0.001$ \\
\hline Weight kg & $4.47 \pm 0.55$ & $4.54 \pm 0.77$ & $0.07(-0.18-0.32)$ & 0.58 \\
\hline Weight centiles & $47.69 \pm 27.36$ & $25.48 \pm 21.36$ & $-22.20(-31.53--12.88)$ & $<0.001$ \\
\hline $\mathrm{BMI} \mathrm{kg} \cdot \mathrm{m}^{-2}$ & $14.9 \pm 1.2$ & $14.8 \pm 1.4$ & $-0.1(-0.6-0.4)$ & 0.68 \\
\hline
\end{tabular}

Data are presented as $\mathrm{n}$ or mean $\pm \mathrm{SD}$, unless otherwise stated. Centiles were calculated based on the growth charts of the Centers for Disease Control and Prevention. BMI: body mass index. ${ }^{\#}$ : t-test or Chi-squared test, as appropriate.

(detailed results are provided in the online supplementary material; see also online supplementary table S4 and figures S1-S4). Analysis in all children with at least two valid measurements confirmed results; mean \pm SD LCI was $7.12 \pm 1.00$ in 47 infants with CF compared to $6.79 \pm 0.42$ in 56 healthy infants (mean difference 0.33 , 95\% CI $0.04-0.63$; $\mathrm{p}=0.025$; online supplementary figure S5; the appropriate results for FRC are displayed in online supplementary figure S6).

\section{Tidal breathing parameters}

Five tidal breathing measurements had to be excluded in infants with CF due to insufficient breathing quality, leaving 48 tidal measurements for analysis in CF compared to 57 in healthy infants. Infants with CF showed significantly higher peak tidal inspiratory flow (mean difference $5.34 \mathrm{~mL}$, 95\% CI 1.18 $9.51 \mathrm{~mL} ; \mathrm{p}=0.012$; table 3), but lower $t \mathrm{PTEF} / \mathrm{tE}$ compared to healthy infants (mean difference $-5.49 \mathrm{~mL}$, 95\% CI $-9.74--1.24 \mathrm{~mL} ; \mathrm{p}=0.012$; table 3 and figure 3 ). The statistical significance of the results did

\section{TABLE 2 Clinical characteristics of infants with cystic fibrosis}

$\begin{array}{lc}\text { Subjects } \mathbf{n} & 53 \\ \text { Respiratory symptoms }{ }^{\#} \text { prior to lung function measurements } & 3(6) \\ \text { With antibiotic treatment } & 1(2) \\ \text { Without antibiotic treatment } & 2(4) \\ \text { Inhalation therapy }{ }^{\|} & 24(45) \\ \quad \text { Started prior to lung function measurement } & 20(38) \\ \text { Started on day of lung function measurement } & 4(8) \\ \text { Antibiotic use } & 9(17) \\ \text { Prior to lung function measurement } & 5(9) \\ \text { At lung function measurement } & 4(8) \\ \text { Indication } & 1(2) \\ \quad \text { Respiratory symptoms prior to lung function } & 7(13) \\ \quad \text { Asymptomatic colonisation } & 1(2) \\ \quad \text { Other (volvulus/ileus) } & \\ \text { CFTR mutation } \$ & 33(62) \\ \text { No residual CFTR function (class I and/or II) } & 9(17) \\ \text { Residual CFTR function (class I/II and III-VI) } & 11(21) \\ \text { Unknown CFTR function } & \end{array}$

Data are presented as $\mathrm{n}(\%)$, unless otherwise stated. CFTR: cystic fibrosis transmembrane conductance regulator. " : any symptoms suggesting respiratory tract infection and/or signs of exacerbation prior to lung function measurement (the presence of respiratory symptoms at lung function measurement was an exclusion criteria); ๆ: beginning of regular inhalation therapy with salbutamol and isotonic saline prior to or at date of lung function measurement (in this case, first inhalation after lung function measurement); ${ }^{+}$: application of antibiotics prior to or at date of lung function measurement; ${ }^{\S}$ : cystic fibrosis infants were grouped into 1) no residual CFTR function (two known copies of class I and/or II mutations), 2) residual CFTR function (at least one mutation with residual CFTR function (class I or II mutation) plus one other mutation), and 3) unknown CFTR function (at least one mutation not classified or unknown mutation class); however, all children in group 3) had two copies of disease-causing mutations. 


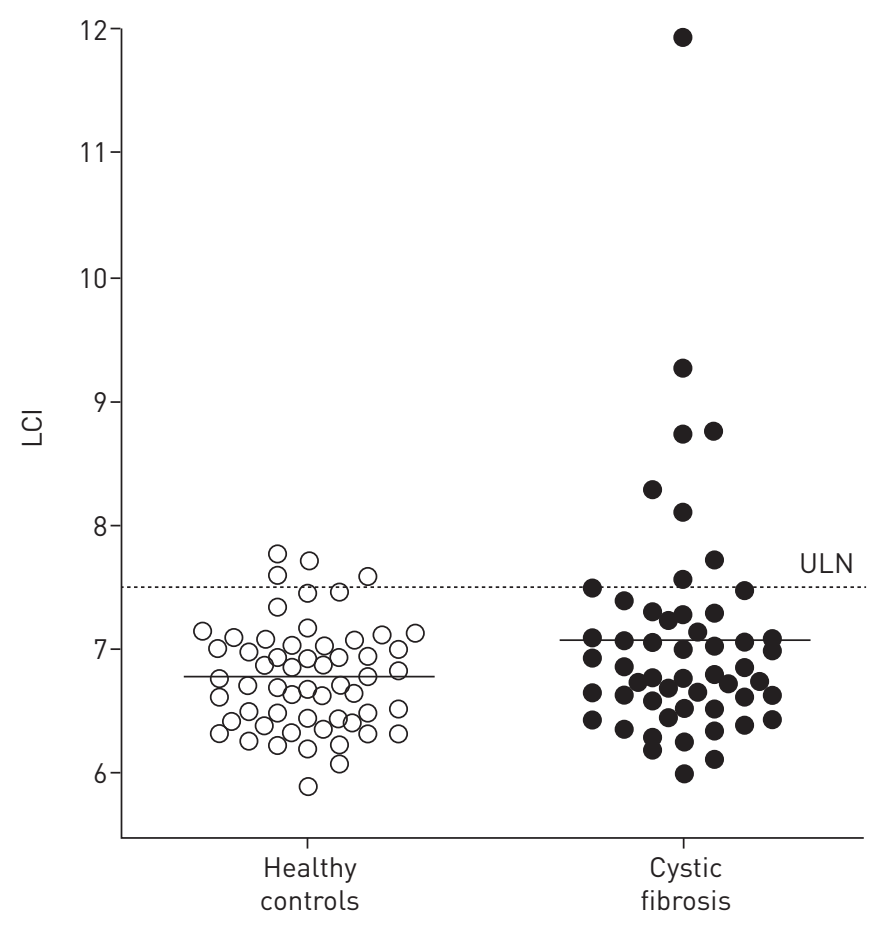

FIGURE 1 Lung clearance index (LCI) and group means of sulfur hexafluoride multiple-breath washout measurement in 57 healthy control infants and 53 infants with cystic fibrosis. ULN: upper limit of normal (mean $\mathrm{LCl}+1.64$ SD from healthy controls).

not change after adjustment for tidal volume or body size. All the other tidal breathing parameters such as tidal volume (tidal volume $\cdot \mathrm{kg}^{-1}$ ) and respiratory rate showed no difference between the two groups (table 3), including after adjustment for age or body size.

Regulatory mechanism of breathing

A post hoc approach was used to assess whether potential factors determining end-expiratory lung volume (FRC) and tidal volume differ between CF and healthy infants. We assumed that body size could be one of the main determinants. Based on previous findings of restricted "freedom of breathing" in preterm infants [30], we examined the association between weight and FRC as well as tidal volume separately in CF and

TABLE 3 Lung function parameters in infants with cystic fibrosis (CF) and healthy controls

Healthy controls

CF

Mean difference

p-value (95\% Cl)

\begin{tabular}{lcccc}
\hline $\begin{array}{l}\text { Multiple-breath washout } \\
\text { Subjects }\end{array}$ & 57 & 53 & & \\
LCl & $6.78 \pm 0.43$ & $7.08 \pm 0.96$ & $0.30(0.02-0.58)$ & 0.034 \\
FRC $\mathrm{mL}$ & $112.3 \pm 15.5$ & $126.7 \pm 20.3$ & $14.5(7.7-21.3)$ & $<0.001$ \\
$\quad$ FRC per body weight $\mathrm{mL} \cdot \mathrm{kg}^{-1}$ & $25.4 \pm 3.9$ & $28.3 \pm 4.3$ & $2.9(1.3-4.4)$ & $<0.001$ \\
Tidal breathing parameter & & & & \\
$\quad$ Subjects & 57 & 48 & & 0.27 \\
Tidal volume $\mathrm{mL} \cdot \mathrm{kg}^{-1}$ & $7.32 \pm 1.05$ & $7.57 \pm 1.17$ & $0.24(-0.19-0.67)$ & 0.75 \\
Respiratory rate $\mathrm{min}^{-1}$ & $43.2 \pm 9.7$ & $42.6 \pm 8.9$ & $-0.6(-4.2-3.1)$ & 0.012 \\
Peak inspiratory flow $\mathrm{mL} \cdot \mathrm{s}^{-1}$ & $71.0 \pm 10.6$ & $76.3 \pm 10.8$ & $5.3(1.2-9.5)$ & 0.29 \\
Peak expiratory flow $\mathrm{mL} \cdot \mathrm{s}^{-1}$ & $59.4 \pm 13.7$ & $62.2 \pm 13.2$ & $2.8(-2.4-8.0)$ & 0.012 \\
tPTEF/tE \% & $39.1 \pm 11.5$ & $33.6 \pm 10.3$ & $-5.5(-9.7--1.2)$ & \\
\hline
\end{tabular}

Data are presented as $\mathrm{n}$ or mean $\pm \mathrm{SD}$, unless otherwise stated, and compared using t-tests. $\mathrm{LCl}$ : lung clearance index; FRC: functional residual capacity; $t$ PTEF: time to peak tidal expiratory flow; $t$ : expiratory time. " : washout measurements analysed in WBreath (ndd Medizintechnik, Zurich, Switzerland). 


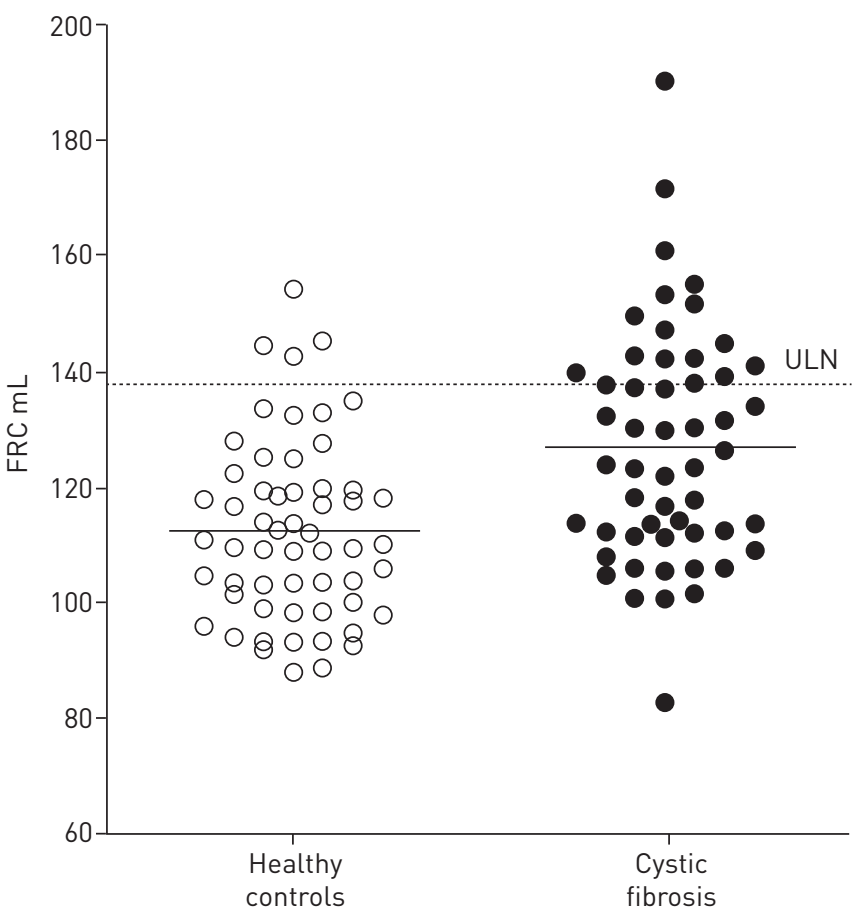

FIGURE 2 Functional residual capacity (FRC) and group means of sulfur hexafluoride multiple-breath washout measurement in 57 healthy infants and 53 infants with cystic fibrosis. ULN: upper limit of normal (mean healthy+1.64 SD from healthy controls).

healthy infants. We found a stronger correlation of weight with FRC in infants with $\mathrm{CF}\left(\mathrm{R}^{2} 40 \%\right.$ in $\mathrm{CF}$ subjects compared to $7 \%$ in healthy infants; figure 4 ), as well as with tidal volume (48\% in CF subjects and $20 \%$ in healthy infants) (online supplementary figure S7), suggesting that stronger regulatory mechanisms take place in infants with CF compared to healthy controls. These differences remained significant after adjustment for age. For further details, see the online supplementary material.

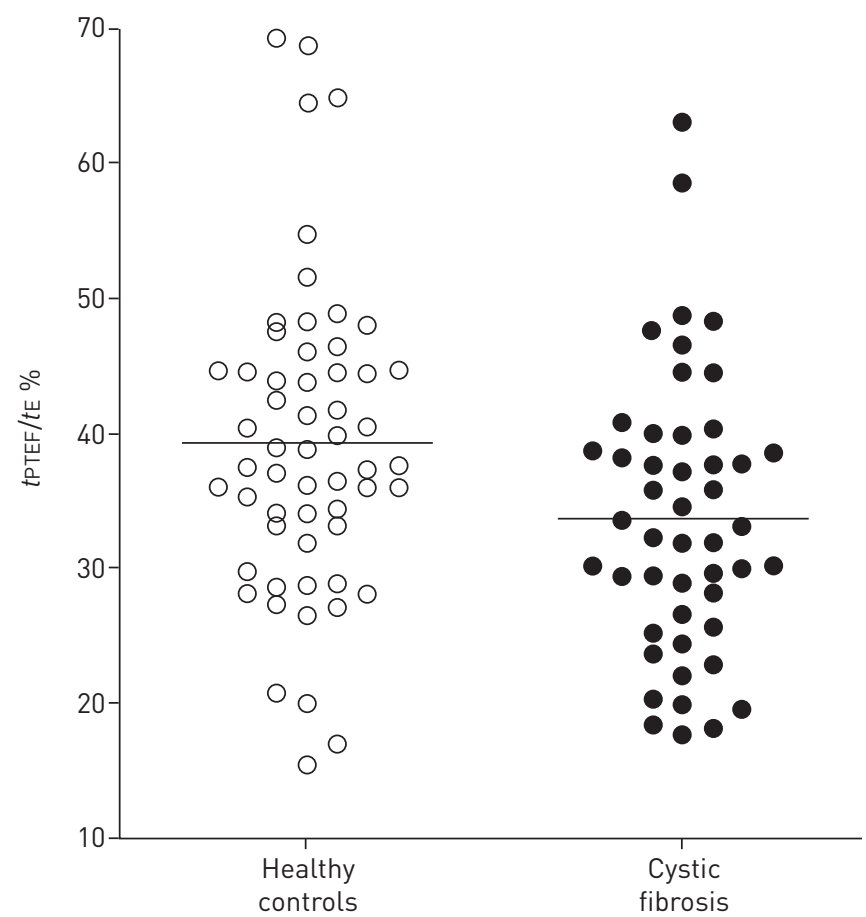

FIGURE 3 Time to peak tidal expiratory flow (tPTEF)/expiratory time ( $t E$ ) ratio of tidal breathing measurement in 57 healthy control infants and 48 infants with cystic fibrosis. Solid lines represent group means. 

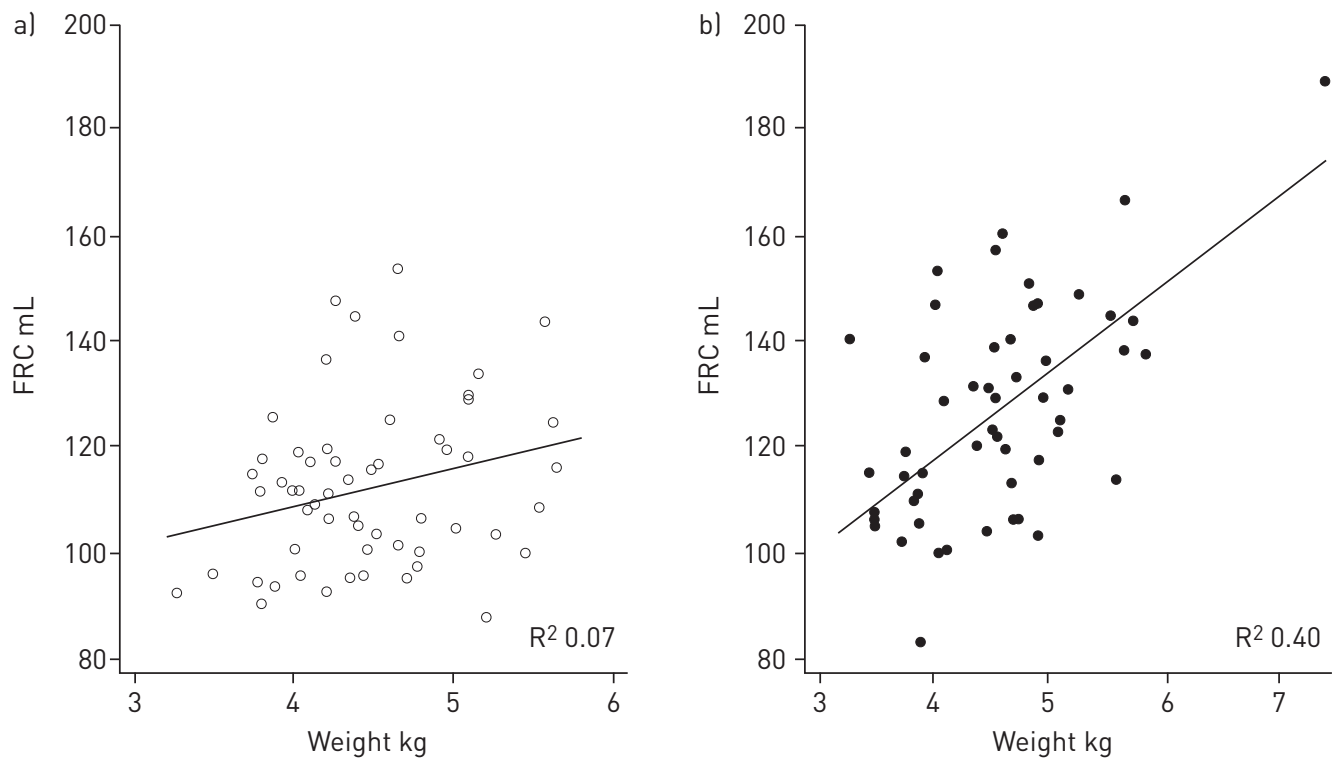

FIGURE 4 Functional residual capacity (FRC) versus weight at lung function assessment for a) healthy infants and b) infants with cystic fibrosis (CF). Lines represent the regression line with a coefficient of correlation of 0.26 for FRC in healthy infants and 0.63 in infants with CF.

Factors associated with elevated lung clearance index

In univariable regression analysis FRC was positively associated with LCI (online supplementary table S5). In addition, peak tidal inspiratory and expiratory flow were positively associated with LCI, while $t \mathrm{PTEF} / t \mathrm{E}$ was inversely associated with LCI (online supplementary table S5). In the multivariable model adjusted for age, body size and sex, only FRC, peak expiratory flow and $t \mathrm{PTEF} / \mathrm{tE}$ ratio remained significantly associated with LCI, and explained $19 \%$ of its variability.

Associations between lung function parameters and clinical data

We did not find any association between LCI, FRC and tidal breathing parameters and clinical data such as respiratory symptoms, use of antibiotics, inhalation therapy and CF transmembrane conductance regulator mutations (table 4; data for tidal breathing parameters are not shown). In a multivariable model adapted for weight and sex, the subgroup taking antibiotics and/or having respiratory symptoms $(\mathrm{n}=11)$ had

TABLE 4 Associations between lung function parameters and clinical data in infants with cystic fibrosis

\begin{tabular}{|c|c|c|c|c|}
\hline & \multicolumn{2}{|c|}{ Multivariable model ${ }^{\#}$} & \multicolumn{2}{|c|}{ Multivariable model ${ }^{\pi}$} \\
\hline & $\boldsymbol{\beta}(95 \% \mathrm{CI})$ & p-value & $\beta(95 \% \mathrm{Cl})$ & p-value \\
\hline \multicolumn{5}{|l|}{ LCI } \\
\hline Respiratory symptoms and/or antibiotic use & $0.93(0.37-1.50)$ & $0.002^{\S}$ & $1.1(0.50-1.71)$ & $0.001^{\S}$ \\
\hline Inhalation therapy & $0.22(-0.27-0.71)$ & 0.378 & $-0.08(-0.57-0.41)$ & 0.737 \\
\hline Known CFTR residual function ${ }^{+}$ & $-0.09(-0.80-0.61)$ & 0.787 & $-0.53(-1.21-0.14)$ & 0.120 \\
\hline \multicolumn{5}{|l|}{ FRC } \\
\hline Respiratory symptoms and/or antibiotic use & $0.002(-0.01-0.01)$ & 0.735 & $0.002(-0.01-0.01)$ & 0.783 \\
\hline Inhalation therapy & $0.004(-0.01-0.01)$ & 0.34 & $0.001(-0.01-0.01)$ & 0.795 \\
\hline Known CFTR residual function ${ }^{+}$ & $0.001(-0.01-0.01)$ & 0.92 & $-0.001(-0.02-0.01)$ & 0.85 \\
\hline
\end{tabular}

Results from the linear regression models assessing the association between lung function parameters and clinical data. Per unit change in a given clinical variable, lung clearance index (LCI) and functional residual capacity (FRC) changes by the effect size of the regression coefficient $\beta .{ }^{*}$ : adjusted for weight and sex; ": adjusted for weight and sex and all other outcomes (respiratory symptoms and/or antibiotic use, inhalation therapy, cystic fibrosis transmembrane conductance regulator (CFTR) mutations); ${ }^{+}$: the baseline was defined as "no residual CFTR function" (for definition, see table 2; 11 infants were summarised as unknown CFTR function showing no significant association with lung function, data not shown); ${ }^{\S}$ : after exclusion of the outlier (see online supplementary figure S8), there was no longer a significant association. 
higher LCI values compared to the subgroup without antibiotics/respiratory symptoms $(n=42)(\beta=0.93,95 \%$ CI $0.37-1.50 ; \mathrm{p}=0.002$; table 4 ). This was due to one outlier, as shown in online supplementary figure S8. After exclusion of this patient the difference was no longer significant (table 4). In the whole population, differences in lung function parameters between infants with CF and healthy infants were independent of the presence of respiratory symptoms and/or use of antibiotics (data not shown).

\section{Discussion}

\section{Summary}

This is the first study showing that abnormal lung function may be present as early as 8 weeks of age in infants with $\mathrm{CF}$, independent of clinical symptoms and previous therapeutic approaches. Functional deficits of early CF lung disease are characterised by decreased ventilation distribution efficiency, elevated end-expiratory lung volumes and altered tidal flows. As early infancy is a pivotal period of reversible airway pathology, this suggests the potential usefulness of noninvasive, relatively simple and accessible lung function measurements, such as MBW, as an outcome for monitoring lung disease and treatment effects. Furthermore, it highlights that abnormal lung function exists in almost half of infants with CF shortly after birth, representing an important future target group to delay disease progression and improve its outcome.

\section{Comparison with the literature}

To our best knowledge, this is the first study on LCI and tidal breathing parameters in infants with CF aged $<3$ months. In older infants and toddlers, several studies have reported elevated LCI, abnormal forced flows and hyperinflation despite early diagnosis by newborn screening and protocol-driven treatment in specialised CF clinics [13, 15-18, 31,32]. Most results come from two large cohorts, the London Cystic Fibrosis Collaboration (LCFC) and the Australian Respiratory Early Surveillance Team for Cystic Fibrosis (AREST-CF) [33]. In the LCFC, comparable to our study, infants with CF had elevated LCI and FRC and reduced forced expiratory volumes $[17,18]$. Mean difference of LCI $(0.46)$ between groups was similar, while prevalence of abnormal LCI was higher (27\% versus $19 \%$ in our study) in the LCFC cohort when using the same ULN (1.64 z-scores). Infants in that study were slightly older ( 3 months of age), sedated and MBW measurements were performed using a customised mass spectrometer, which is no longer available [9]. The AREST-CF used the same MBW setup as we did and showed comparable results for LCI in CF $(7.0 \pm 0.5)$ and prevalence of abnormal LCI (16\%) compared to our cohort [13]. However, the AREST-CF study did not include healthy controls and infants were sedated for the measurements. Furthermore, in the original publication LCI data was analysed using the original software algorithm; in a subsequent analysis using the improved algorithm [25], three more children had abnormal LCI, resulting in increased prevalence of $22 \%$ for abnormal LCI [32]. However, comparison to normative data from different centres using different protocols or sedation probably influences the ULN [34]. In addition, higher LCI values were found in infants and toddlers with CF aged between 1 and 2 years compared to healthy peers, indicating ongoing early lung disease $[15,16,31]$. Different set-ups and analysis methods make it difficult to compare results with regards to the possibility of using MBW to monitor disease progression.

\section{Strengths and limitations}

The major strength of this study is the very young age of infants and a contemporary healthy control group of an equal group size and matching body dimensions. Body size is generally considered a more important confounder than age. Despite natural breathing, intratest variability of lung function indices was low. We were able to replicate our findings in several sensitivity analyses, such as using only data from the wash-in. Compared to other studies, ours is the only one performing lung function measurements without the use of sedation, possibly better reflecting natural regulation of lung physiology. Sedation alters respiratory control and may blur abnormality in tidal breathing indices. Indeed, sedation decreases FRC and increases LCI [21]. Whether this is due to a decrease in thorax wall stability and/or change in breathing pattern is unclear [35]. Chloral hydrate, which is mostly used nowadays for sedation in infants, may also influence tidal volumes [36], blood pressure and carbon dioxide respiration [37]. Since natural breathing regulation is altered by sedation $[36,37]$ and probably depends on underlying pathophysiology, possible breathing pattern abnormalities might be over- or underestimated. Findings from similar MBW studies with and without the use of chloral hydrate in infants with bronchopulmonary dysplasia strongly support this assumption [30,38]. Furthermore, chloral hydrate is restricted to research applications for infant lung function (in the USA), and other sedatives such as propofol may lead to excessive sedation [39, 40] and changes in breathing pattern [21]. However, while measurements in natural sleep are feasible during the first weeks of life, this becomes more challenging in older infants, who then require sedation. Comparability of results might then be again limited.

A limitation of our study might be the cross-sectional study design, as lung function in early infancy is potentially dynamic and might be related to infections and therapy. Clearly, repeated measurements on a 
regular basis would be needed to fully understand dynamics of altered lung function. We applied a single ULN value for LCI since the LCI showed no age dependency over the narrow age range in our healthy infant study population (online supplementary figure S9). Based on international recommendations, we chose a $90 \%$ prediction interval for the ULN of washout parameters (mean+1.64 SD) instead of a 95\% prediction interval (mean+1.96 SD) [29]. However, we need to be aware that this lowers the ULN, resulting in more patients with elevated washout parameters. In addition, we included a small number of infants with only one valid measurement. Results were verified in an analysis excluding those children (sensitivity analysis iii in the online supplementary material). Additionally, since this study was explanatory and not conducted to primarily identify clinical risk factors associated with elevated LCI, we can only speculate about possible underlying physiological mechanisms.

\section{Physiological considerations}

Our data suggest that impaired lung function may be already present shortly after birth. Patchy mucus distribution and/or airway wall thickening in the lung periphery seem to lead to hyperinflation [2] and increased ventilation inhomogeneity. Reduced $t \mathrm{PTEF} / \mathrm{tE}$ ratios either indicate altered flow properties due to airway obstruction or changes in respiratory drive aiming to prevent airway collapse. This leads to a restricted breathing pattern with poor physiological fluctuation, at least in natural sleep, which itself may further contribute to mucus plugging and increased ventilation heterogeneity. This hypothesis is clearly supported by the greater association between tidal volume and FRC with body size in infants with CF than in healthy infants. Similar observations have been made in former preterm infants compared to term-born infants, also assessed in natural sleep [30].

\section{Implications for clinical practice and scientific relevance}

The predictive value of abnormal lung function, e.g. for infection or structural lung damage seems rather poor in infants with CF $[13,15,32]$. Yet early abnormal lung function cannot be ignored as studies in older children show that abnormal lung function may become irreversible [41] and tracks into preschool [42] and school age [14]. Thus, infant lung function using MBW could be regarded as a potential standalone biomarker to assess the response to therapy and may be used as a study end-point for early interventional studies attempting to preserve lung function rather than slow its decline [18].

\section{Conclusions}

Abnormal lung function as measured by LCI and FRC is present in $>40 \%$ of 8 -week-old infants with CF. Despite early diagnosis and therapy, this apparently inherent characteristic of CF lung disease cannot yet be fully prevented. Our findings should alert clinicians not to rely only on absence of respiratory symptoms in infants with CF. Our data were obtained using commercially available MBW equipment and during natural sleep without sedation. Both factors are important for clinical practicability and more widespread use. This highlights the potential usefulness of MBW as an outcome for monitoring disease and disease-modifying studies in this age group.

\section{Acknowledgements}

The authors would like to thank all the children and their families for their participation in the study. The authors would like to thank Ines Mack (Basel) and Giulia Cangiano (Rome) for lung function measurements and valuable input. We express our gratitude to Sandra Luescher, Monika Graf, Sharon Schmid and Gisela Wirz (Bern) for their valuable work and support. The authors would like to thank the Swiss Cystic Fibrosis Newborn Screening Group (Juerg Barben, St Gallen; Constance Barazzone, Geneva; Matthias R. Baumgartner, Zurich; Sabina Gallati, Bern; Ralph Fingerhut, Zurich; Claudia E. Kuehni, Bern; Peter Eng, Aarau; Gaudenz Hafen, Lausanne; Jürg Hammer, Basel; Corina S. Ruegg, Bern; Barbara Schiller, St Gallen; Martin H. Schöni, Bern; Renate Spinas, Zurich; Johannes Spalinger, Lucerne; and Toni Torresani, Zurich) for their valuable work.

Authors contributions are as follows. Conception and design of the study: E. Kieninger, S. Yammine and P. Latzin; acquisition of data: E. Kieninger, S. Yammine, I. Korten, P. Anagnostopoulou, F. Singer, U. Frey, A. Mornand, M. Zanolari, I. Rochat, D. Trachsel, D. Mueller-Suter, A. Moeller, C. Casaulta and P. Latzin; analysis and interpretation: E. Kieninger, S. Yammine, I. Korten, F. Singer, C. Casaulta and P. Latzin; drafting the manuscript for important intellectual content: E. Kieninger, S. Yammine, F. Singer, C. Casaulta and P. Latzin; final approval of the manuscript: all authors.

Members of the Swiss Cystic Fibrosis Infant Lung Development (SCILD) cohort, current study group: Juerg Barben (St Gallen), Carmen Casaulta (Bern), Gaudenz Hafen (Lausanne), Elisabeth Kieninger (Bern), Insa Korten (Bern), Philipp Latzin (Bern), Alexander Moeller (Zurich), Anne Mornand (Geneva), Dominik Mueller-Suter (Aarau), Nicolas Regamey (Lucerne), Isabelle Rochat (Lausanne), Florian Singer (Zurich), Daniel Trachsel (Basel), Sophie Yammine (Bern) and Maura Zanolari (Bellinzona).

Members of the Basel-Bern Infant Lung Development (BILD) cohort, current study group: Pinelopi Anagnostopoulou (Bern), Anne-Christianne Kentgens (Bern), Barbara Egger (Basel), Urs Frey (Basel), Oliver Fuchs (Bern), Claudia Kuehni (Bern), Philipp Latzin (Bern), Elena Proietti (Bern), Anne Schmidt (Bern) and Jakob Usemann (Basel). 


\section{References}

1 Castellani C, Massie J, Sontag M, et al. Newborn screening for cystic fibrosis. Lancet Respir Med 2016; 4: 653-661.

2 Sly PD, Brennan S, Gangell C, et al. Lung disease at diagnosis in infants with cystic fibrosis detected by newborn screening. Am J Respir Crit Care Med 2009; 180: 146-152.

3 Mott LS, Park J, Murray CP, et al. Progression of early structural lung disease in young children with cystic fibrosis assessed using CT. Thorax 2012; 67: 509-516.

4 McKone EF, Borowitz D, Drevinek P, et al. Long-term safety and efficacy of ivacaftor in patients with cystic fibrosis who have the Gly551Asp-CFTR mutation: a phase 3, open-label extension study (PERSIST). Lancet Respir Med 2014; 2: 902-910.

5 Yammine S, Singer F, Latzin P. CFTR modulation for young children with cystic fibrosis. Lancet Respir Med 2016; 4: 84-85.

6 Sermet-Gaudelus I, Mayell SJ, Southern KW. Guidelines on the early management of infants diagnosed with cystic fibrosis following newborn screening. J Cyst Fibros 2010; 9: 323-329.

7 Stick SM, Sly PD. Exciting new clinical trials in cystic fibrosis: infants need not apply. Am J Respir Crit Care Med 2011; 183: 1577-1578.

8 Ramsey KA, Ranganathan S. Interpretation of lung function in infants and young children with cystic fibrosis Respirology 2014; 19: 792-799.

9 Robinson PD, Latzin P, Verbanck S, et al. Consensus statement for inert gas washout measurement using multiple- and single-breath tests. Eur Respir J 2013; 41: 507-522.

10 Gustafsson PM, Aurora P, Lindblad A. Evaluation of ventilation maldistribution as an early indicator of lung disease in children with cystic fibrosis. Eur Respir J 2003; 22: 972-979.

11 Gustafsson PM, De Jong PA, Tiddens HA, et al. Multiple-breath inert gas washout and spirometry versus structural lung disease in cystic fibrosis. Thorax 2008; 63: 129-134.

12 Singer F, Kieninger E, Abbas C, et al. Practicability of nitrogen multiple-breath washout measurements in a pediatric cystic fibrosis outpatient setting. Pediatr Pulmonol 2013; 48: 739-746.

13 Hall GL, Logie KM, Parsons F, et al. Air trapping on chest CT is associated with worse ventilation distribution in infants with cystic fibrosis diagnosed following newborn screening. PLoS One 2011; 6: e23932.

14 Kieninger E, Singer F, Fuchs O, et al. Long-term course of lung clearance index between infancy and school-age in cystic fibrosis subjects. J Cyst Fibros 2011; 10: 487-490.

15 Belessis Y, Dixon B, Hawkins G, et al. Early cystic fibrosis lung disease detected by bronchoalveolar lavage and lung clearance index. Am J Respir Crit Care Med 2012; 185: 862-873.

16 Stahl M, Joachim C, Blessing K, et al. Multiple breath washout is feasible in the clinical setting and detects abnormal lung function in infants and young children with cystic fibrosis. Respiration 2014; 87: $357-363$.

17 Hoo AF, Thia LP, Nguyen TT, et al. Lung function is abnormal in 3-month-old infants with cystic fibrosis diagnosed by newborn screening. Thorax 2012; 67: 874-881.

18 Nguyen TT, Thia LP, Hoo AF, et al. Evolution of lung function during the first year of life in newborn screened cystic fibrosis infants. Thorax 2014; 69: 910-917.

19 Lum S, Gustafsson P, Ljungberg H, et al. Early detection of cystic fibrosis lung disease: multiple-breath washout versus raised volume tests. Thorax 2007; 62: 341-347.

20 Mallol J, Sly PD. Effect of chloral hydrate on arterial oxygen saturation in wheezy infants. Pediatr Pulmonol 1988; 5: 96-99.

21 von Ungern-Sternberg BS, Frei FJ, Hammer J, et al. Impact of depth of propofol anaesthesia on functional residual capacity and ventilation distribution in healthy preschool children. Br J Anaesth 2007; 98: 503-508.

22 Torresani T, Fingerhut R, Rueegg CS, et al. Newborn screening for cystic fibrosis in Switzerland - consequences after analysis of a 4 months pilot study. J Cyst Fibros 2013; 12: 667-674.

23 Mika M, Korten I, Qi W, et al. The nasal microbiota in infants with cystic fibrosis in the first year of life: a prospective cohort study. Lancet Respir Med 2016; 4: 627-635.

24 Fuchs O, Latzin P, Kuehni CE, et al. Cohort profile: the Bern infant lung development cohort. Int J Epidemiol 2012; 41: 366-376

25 Anagnostopoulou P, Yammine S, Schmidt A, et al. False normal lung clearance index in infants with cystic fibrosis due to software algorithms. Pediatr Pulmonol 2015; 50: 970-977.

26 Fuchs O, Latzin P, Thamrin C, et al. Normative data for lung function and exhaled nitric oxide in unsedated healthy infants. Eur Respir J 2011; 37: 1208-1216.

27 Latzin P, Sauteur L, Thamrin C, et al. Optimized temperature and deadspace correction improve analysis of multiple breath washout measurements by ultrasonic flowmeter in infants. Pediatr Pulmonol 2007; 42: 888-897.

28 Anagnostopoulou P, Egger B, Lurà M, et al. Multiple breath washout analysis in infants: quality assessment and recommendations for improvement. Physiol Meas 2016; 37: L1-L15.

29 Quanjer PH, Stanojevic S, Cole TJ, et al. Multi-ethnic reference values for spirometry for the 3-95-yr age range: the global lung function 2012 equations. Eur Respir J 2012; 40: 1324-1343.

30 Latzin P, Roth S, Thamrin C, et al. Lung volume, breathing pattern and ventilation inhomogeneity in preterm and term infants. PLoS One 2009; 4: e4635.

31 Stahl M, Wielpütz MO, Graeber SY, et al. Comparison of lung clearance index and magnetic resonance imaging for assessment of lung disease in children with cystic fibrosis. Am J Respir Crit Care Med 2017; 195: 349-359.

32 Ramsey KA, Rosenow T, Turkovic L, et al. Lung clearance index and structural lung disease on computed tomography in early cystic fibrosis. Am J Respir Crit Care Med 2016; 193: 60-67.

33 Bush A, Sly PD. Evolution of cystic fibrosis lung function in the early years. Curr Opin Pulm Med 2015; 21: $602-608$.

34 Stocks J, Modi N, Tepper R. Need for healthy control subjects when assessing lung function in infants with respiratory disease. Am J Respir Crit Care Med 2010; 182: 1340-1342.

35 Yammine S, Singer F, Gustafsson P, et al. Impact of different breathing protocols on multiple-breath washout outcomes in children. J Cyst Fibros 2014; 13: 190-197.

36 Turner DJ, Morgan SE, Landau LI, et al. Methodological aspects of flow-volume studies in infants. Pediatr Pulmonol 1990; 8: 289-293. 
37 Wilson S. Chloral hydrate and its effects on multiple physiological parameters in young children: a dose-response study. Pediatr Dent 1992; 14: 171-177.

38 Schmalisch G, Wilitzki S, Roehr CC, et al. Development of lung function in very low birth weight infants with or without bronchopulmonary dysplasia: longitudinal assessment during the first 15 months of corrected age. BMC Pediatr 2012; 12: 37.

39 Ren CL, Robinson P, Ranganathan S. Chloral hydrate sedation for infant pulmonary function testing. Pediatr Pulmonol 2014; 49: 1251-1252.

40 Callahan P, Pinto SJ, Kurland G, et al. Dexmedetomidine for infant pulmonary function testing. Pediatr Pulmonol 2015; 50: 150-154.

41 Birket SE, Chu KK, Liu L, et al. A functional anatomic defect of the cystic fibrosis airway. Am J Respir Crit Care Med 2014; 190: 421-432.

42 Aurora P, Stanojevic S, Wade A, et al. Lung clearance index at 4 years predicts subsequent lung function in children with cystic fibrosis. Am J Respir Crit Care Med 2011; 183: 752-758. 